\title{
Interações medicamentosas entre pacientes com HIV/AIDS
}

\author{
Drug interactions between patients with HIV/AIDS \\ Interacciones medicamentosas entre pacientes con VIH/SIDA
}

Recebido: 04/11/2021 | Revisado: 11/11/2021 | Aceito: 15/11/2021 | Publicado: 22/11/2021

Isabelle Aparecida de Sousa Bernardes
ORCID: https://orcid.org/0000-0002-5147-2222
Universidade do Estado de Minas Gerais, Brasil
E-mail: isabelleasousa@ @otmail.com
Jhenifer Alves de Araújo
ORCID: https://orcid.org/0000-0002-7777-8318
Universidade do Estado de Minas Gerais, Brasil
E-mail: jheni.araujo1998@ gmail.com
Eduardo Nogueria Cortez
ORCID: https://orcid.org/0000-0003-4974-1451
Universidade do Estado de Minas Gerais, Brasil
E-mail: eduardonogueiracortez@ gmail.com
Maíla Martins Oliveira
ORCID: https://orcid.org/0000-0002-0289-2378
Universidade do Estado de Minas Gerais, Brasil
E-mail: maila.oliveira @ uemg.br
Marlene Alves Ferreira
ORCID: https://orcid.org/0000-0001-5348-0886
Secretaria Municipal de Saúde, Brasil
E-mail: alvesferreiramarlene8@ gmail.com
Deborah Franscielle da Fonseca
ORCID: https://orcid.org/0000-0001-6001-2837
Universidade Federal de São João del Rei, Brasil
E-mail: deborahfonseca@ @hotmail.com
Heuler Souza Andrade
ORCID: https://orcid.org/0000-0001-8552-3131
Universidade do Estado de Minas Gerais, Brasil
E-mail: heuler.andrade@uemg.br

\section{Resumo}

Objetivo: Analisar por meio de evidências científicas a ocorrência de interações droga-droga em pacientes com diagnóstico de HIV/AIDS. Metodologia: Trata-se de uma revisão integrativa. A coleta de dados ocorreu através da Base Virtual de Saúde realizada no dia 22 de novembro de 2020. Assim sendo, utilizou-se o aplicativo Rayyan para otimizar a seleção dos artigos e a matriz de síntese foi o método para realização da análise dos dados. Resultados: Foi possível identificar que poucos estudos são realizados com a população idosa que apresenta o diagnóstico de HIV/AIDS. Além disso, observou-se que as principais interações medicamentosas ocorrem entre antirretrovirais e medicamentos inibidores da bomba de prótons. Conclusão: A ocorrência de interações medicamentosas é frequente em pacientes com diagnóstico de HIV/AIDS, principalmente em idosos. Sendo assim, é fundamental que sejam realizadas novas pesquisas sobre essa temática, visando a formulação de estratégias para minimizar as interações droga-droga e suas consequências para o paciente.

Palavras-chave: Interações medicamentosas; Infecção por HIV; Enfermagem.

\begin{abstract}
Objective: To analyze through scientific evidence the occurrence of drug-drug interactions in patients diagnosed with HIV/AIDS. Methodology: This is an integrative review. Data collection took place through the Virtual Health Database held on November 22, 2020. Therefore, the Rayyan application was used to optimize the selection of articles and the synthesis matrix was the method for carrying out the data analysis. Results: It was possible to identify that few studies are carried out with the elderly population that has a diagnosis of HIV/AIDS. Furthermore, it was observed that the main drug interactions occur between antiretrovirals and proton pump inhibitor drugs. Conclusion: The occurrence of drug interactions is frequent in patients diagnosed with HIV/AIDS, especially in the elderly. Therefore, it is essential that further research be carried out on this topic, aiming to formulate strategies to minimize drug-drug interactions and their consequences for the patient.
\end{abstract}

Keywords: Drug interactions; HIV Infections; Nursing. 


\begin{abstract}
Resumen
Objetivo: Analizar a través de evidencia científica la ocurrencia de interacciones fármaco-fármaco en pacientes diagnosticados de VIH / SIDA. Metodología: Se trata de una revisión integradora. La recolección de datos se realizó a través de la Base de Datos Virtual en Salud realizada el 22 de noviembre de 2020. Por lo tanto, se utilizó la aplicación Rayyan para optimizar la selección de artículos y la matriz de síntesis fue el método para realizar el análisis de datos. Resultados: Se pudo identificar que se realizan pocos estudios con la población anciana que tiene un diagnóstico de VIH / SIDA. Además, se observó que las principales interacciones farmacológicas se producen entre los antirretrovirales y los fármacos inhibidores de la bomba de protones. Conclusión: La ocurrencia de interacciones medicamentosas es frecuente en pacientes diagnosticados de VIH / SIDA, especialmente en ancianos. Por tanto, es fundamental que se realicen más investigaciones sobre este tema, con el objetivo de formular estrategias para minimizar las interacciones fármaco-fármaco y sus consecuencias para el paciente.
\end{abstract}

Palabras clave: Interacciones farmacológicas; Infecciones por VIH; Enfermería.

\title{
1. Introdução
}

A interação medicamentosa (IM) ou interações droga-droga são definidas pela alteração na ação de um medicamento, causada pela administração simultânea ou prévia de outros medicamentos. Isso acontece, pois, a IM pode interferir nas ações farmacocinéticas e/ou farmacodinâmicas um do outro, o que pode resultar em uma eficácia reduzida ou aumentada, conforme a combinação de medicamentos e/ou aumento ou redução da toxicidade (Turgeon \& Michaud, 2016). Conforme a Organização Mundial da Saúde, a IM é a principal causa de morbimortalidade no mundo, dados indicam que anualmente, somente nos Estados Unidos, mais de 2 milhões de pacientes hospitalizados sofrem reações adversas em decorrência de medicamentos e aproximadamente 100.000 óbitos ocorrem por este motivo (Monteiro et al., 2019)

Verifica-se, que os efeitos farmacológicos apresentam a possibilidade de sofrer alteração por meio da presença de um alimento, bebida ou algum agente químico, dentre outros. Esse efeito farmacológico pode ser sinérgico ou antagônico, sendo sinérgico, quando o efeito terapêutico pode ser potencializado e antagônico quando a administração simultânea pode diminuir a eficácia do medicamento utilizado. A IM também pode interferir na absorção, metabolização e na eliminação do medicamento. (Moreira et al., 2017; Monteiro et al., 2015). Logo, as IM possuem a capacidade de elevar os efeitos adversos de determinada medicação, gerar a ineficácia do tratamento e repercutir negativamente na saúde do paciente, acarretando um número elevado de hospitalizações indesejadas, aumentando o custo do tratamento e tendo potencial de gerar o óbito do paciente (Moreira et al., 2017).

A IM pode ser classificada conforme sua gravidade, sendo graves quando oferecem risco à vida ou dano irreversível, implicando em intervenção médica urgente para a redução dos efeitos adversos. São de gravidade moderada quando causam a piora do estado clínico do paciente sendo necessário tratamento adicional, como hospitalização. Quando classificada como leves, os efeitos clínicos são pequenos e a consequência pode ser um desconforto, mas não demanda alterações importantes na terapia (Tavares et al., 2018).

Deste modo, as interações droga-droga refletem um aspecto extremamente complexo na clínica farmacológica, visto que, geralmente, as informações sobre às possíveis interações são produzidas durante o desenvolvimento de um medicamento e não possui eficácia para determinar sua importância clínica. As informações sobre as IM nos seres humanos vêm de modelos experimentais ou estudos em voluntários saudáveis que recebem uma única dose em situações diferentes e desta forma não é retratado o que ocorre no cotidiano dos pacientes (Gervasoni et al., 2019).

Os principais fatores de risco para uma possível IM, incluem: a classe de medicamentos; as prescrições médicas, relacionadas com um número elevado de medicamentos prescritos; a complexidade do quadro clínico; e os fatores associados aos pacientes, sendo eles a idade, patologia coexistente, polifarmácia (Moreira et al., 2017). Cabe destacar, as IM como um dos eventos adversos mais frequentes durante o atendimento ao paciente e apresenta a capacidade de resultar em abandono do tratamento, elevar o número de hospitalização, além de induzir reações adversas, reduzir a eficácia do medicamento e isso acontece principalmente em sujeitos em polifarmácia (Santos et al., 2016). 
A polifarmácia, isto é, o uso simultâneo de vários medicamentos, tem aumentado os impasses no cuidado de pessoas com comorbidades (Hasler et al., 2015). Em suma, a polifarmácia ou polimedicação estão relacionadas com a maior complexidade ao combinar um tratamento terapêutico (Bastida et al., 2017).Portanto, pode causar a redução na adesão ao tratamento e reações adversas conforme os medicamentos utilizados e, quando utilizada com a intenção de elevar a eficácia terapêutica, pode acontecer as IM trazendo prejuízos à saúde, os eventos adversos e sua gravidade associados com a IM se intensificam principalmente pelo uso de um maior número de fármacos, além de fatores individuais, como idade avançada e presença de comorbidades (Kara et al., 2019; Andrade \& Souza, 2018).

Embora as incidências de IM sejam mais altas em idosos, por utilizarem diversos medicamentos, outros indivíduos também podem apresentar este perfil, como no caso dos indivíduos que fazem o uso da terapia antirretroviral (TARV). A polifarmácia neste grupo de pacientes, assim como idade, uso de álcool, drogas ilícitas e lícitas, apresenta a possibilidade de interagir com alguns fármacos antirretrovirais, aumentando a complexidade do manuseio terapêutico e os riscos de uma IM (Popets et al., 2015; Santos et al., 2016).

Os antirretrovirais são medicamentos com a finalidade de tratar as infecções causadas por retrovírus e como profilaxia para doenças oportunistas. A partir do ano de 2017, os medicamentos dolutegravir em associação com tenofovir e lamivudina, são utilizados como o tratamento do Vírus da Imunodeficiência Humana (HIV)/Síndrome da Imunodeficiência Adquirida (AIDS) de primeira escolha no Brasil (Nunes Júnior \& Ciosak, 2018).

Destaca-se, que o HIV, causa efeitos nas células em um curto período e assim uma infecção persistente que gera um quadro clínico de imunodeficiência, correspondendo à AIDS, que atualmente pode ser considerada uma doença crônica que possui tratamento, porém não há cura (Silva et al., 2019; Costa et al., 2018)

A infecção pelo HIV não é possível ser erradicada, porém com o uso da TARV ocorreu uma postergação das consequências do HIV/AIDS e com isso a redução da mortalidade e consequentemente a transformação para uma doença crônica. Ressalta-se que a TARV possui o objetivo de desacelerar a evolução da doença, proporcionando a redução da carga viral a um valor abaixo do limiar de detecção, a estimulação da manutenção da imunidade resultando em aumento da sobrevida dos indivíduos infectados pelo HIV (Silva et al., 2019; Bastida et al., 2017).

Neste cenário a transformação do HIV em uma doença crônica de longo prazo, propiciada pelo uso da TARV pelos pacientes, melhorou o tratamento desses indivíduos e contribuiu para suprimir a replicação do vírus. Logo houve, também, um envelhecimento dessa população infectada que por sua vez foi acometida por doenças crônicas não transmissíveis (DCNT) relacionadas com a idade (Smit et al., 2015).

Assim sendo, o aumento da sobrevida dos pacientes infectados pelo HIV trouxe complicações para os cuidados médicos, uma vez que houve um aumento no número de comorbidades e, portanto, a polifarmácia. Alguns pacientes realizam o uso de algum medicamento simultâneo com a TARV para a reduzir os efeitos colaterais e, simultaneamente, medicamentos para o tratamento de outras comorbidades, o que eleva o risco de eventos adversos, não adesão as prescrições médicas, automedicação, resultando em IM, comprometendo o tratamento do HIV e das comorbidades existentes. (Gervasoni et al., 2019; Siefried, et al., 2018).

Ressalta-se que, os agentes da TARV representam um dos principais grupos com risco potencial para IM, pois os fármacos utilizados podem ser influenciados por isoenzimas, transportadores e estruturas. Conforme, o uso de fármacos concomitantes, como aqueles utilizados no tratamento de DCNT, sua atividade torna-se capaz de ser modificada, resultando em mudanças nos níveis séricos e influenciando os objetivos terapêuticos e a saúde do paciente. (Santos et al., 2016; Osorio et al., 2019).

As IM são crescentes em pacientes infectados pelo HIV, pois apresentam a possibilidade de elevar à toxicidade do medicamento e como consequência a falha do tratamento (Stader et al., 2018). Os principais fatores de risco que elevam o risco 
potencial de IM em pacientes tratados com a TARV são a idade, elevado número de comorbidades, alto número de medicamentos usados pelos pacientes e o uso de inibidores de protease no tratamento com a TARV (Baecke et al., 2017). Segundo Bastida et al. (2017), é necessário a revisão periódica da medicação com a formulação de diretrizes para o uso de drogas em pacientes infectados pelo HIV, com abordagem multidisciplinar, para quando necessário ser alterados os cuidados prestados a estes pacientes de forma integral, em conjunto com todos os profissionais de saúde.

Há a necessidade de identificar os principais problemas relacionados com medicamentos e as prescrições médicas inadequadas ou incompletas. Em virtude disso, torna-se fundamental a capacitação dos profissionais que lidam com a liberação, administração e manuseio da medicação para a análise da prescrição de medicamentos e para atuar em sua administração, além de buscar uma comunicação efetiva e aberta entre farmacêuticos, profissionais de enfermagem e médicos que prescreveram o medicamento, exigindo assim que estes profissionais sejam capacitados busquem conhecimento farmacoterapêutico (Carvalho et al., 2016).

Desta maneira, é fundamental salientar a importância para a segurança do paciente, durante a terapia medicamentosa, a fim de reduzir riscos e falhas durante a administração de medicamentos, além de identificar possíveis interações droga-droga (Antunes et al., 2015).

As IM apresentam capacidade de interferir no tratamento do paciente, além de acarretar prejuízos a saúde do usuário. Assim sendo, percebe-se um possível despreparo dos profissionais de saúde na realização de um atendimento qualificado aos pacientes que apresentam HIV/AIDS e outras comorbidades, apresenta um impacto na identificação das possíveis IM e em seus efeitos indesejáveis para os usuários de determinado serviço de saúde.

Nesta perspectiva, evidencia-se as IM como uma questão crítica durante o atendimento ao paciente, pois elas podem elevar o risco de eventos adversos e aumentar a mortalidade do paciente (Bouzillé et al., 2019).Além disso, poucos estudos são desenvolvidos nesta perspectiva por profissionais de saúde, os quais estão diretamente ligados a rotina de medicamentos dos pacientes e no seu cotidiano possuem papel fundamental em examinar e interferir diante a suspeita de IM, prevenindo principalmente as reações adversas em decorrência da combinação de diversos medicamentos (Santos et al., 2016).

Os profissionais de saúde necessitam de amplo conhecimento sobre os pacientes que apresentam risco de IM. Logo, torna-se fundamental que seja de conhecimento de todos os profissionais de saúde, dentre eles os profissionais que lidam diretamente com medicações e prescrições de medicamentos dos pacientes atendidos em determinado serviço de saúde, as características das drogas utilizadas, a fim de entender quais as possíveis IM diante os medicamentos utilizados. Assim, conhecer a prevalência das IM possibilita a identificação precoce e consequentemente evitar os eventos adversos e possíveis complicações a saúde do paciente, decorrente da combinação de determinados medicamentos, que possam causar a IM.

Nesse sentido, objetivou-se analisar por meio de evidências científicas a ocorrência de interações droga-droga em pacientes com diagnóstico de HIV/AIDS.

\section{Metodologia}

Trata-se de uma revisão integrativa da literatura (RI) realizada entre 2020 e 2021, baseada na seguinte pergunta: "Qual a ocorrência de interações medicamentosas em pacientes com o diagnóstico de HIV/AIDS ao longo do tratamento?".

A RI é um método que visa a investigação, análise crítica e síntese das evidências disponíveis sobre determinado assunto examinado, objetivando a identificação de fragilidades e implementação de intervenções efetivas diante a prestação de cuidados (Sousa et al., 2017).

Por ser baseada na prática baseada em evidências, recomenda-se que a construção da RI, siga as seis etapas: 1) identificação do tema e seleção da questão norteadora da pesquisa; 2) estabelecimento dos critérios de exclusão e inclusão para a realização da busca da literatura; 3) identificação dos estudos e informações que serão obtidas nos estudos selecionados e 
pré-selecionados; 4) avaliação e categorização dos estudos escolhidos; 5) interpretação e análise dos resultados encontrados e 6) apresentação das evidências científicas encontradas(Sousa et al., 2017).

A partir da questão norteadora foi utilizado o acrônimo PICOT na intenção de tornar a busca de dados nas bases eletrônicas eficientes: (P) população alvo; (I) foco da intervenção; (C) compara ou não os tipos de intervenção; (O) resultados das intervenções; (T) influência da intervenção ao longo do tempo (Rodrigues et al., 2020; Joaquim et al., 2018).

Assim sendo, a estratégia de busca ocorreu da seguinte forma: utilização dos descritores controlados contidos nos Descritores em Ciência da Saúde (DEcS) (Tabela 1). Para combinação dos DEcS foi utilizado o operador booleano "AND" para combinação entre os sinônimos foi utilizado "OR”, conforme a técnica PICOT.

Tabela 1: Definição dos DEcS de cada acrônimo retratados por meio da técnica PICOT, 2020-2021.

\begin{tabular}{|c|c|c|c|}
\hline Acrônimo & DEcS & Termos alternativos & Definição \\
\hline $\mathrm{P}$ & $\begin{array}{l}\text { "Infecções por } \\
\text { HIV" }\end{array}$ & $\begin{array}{l}\text { "Coinfecção pelo HIV”; “Coinfecção por HIV”; "Infecções por HTLV- } \\
\text { III”; "Infecções por HTLV-III-LAV”; "Infecções por Virus Linfotrópico T } \\
\text { Humano Tipo III" }\end{array}$ & Pessoas com HIV \\
\hline I & $\begin{array}{l}\text { "Interações } \\
\text { medicamentosas" }\end{array}$ & "Interações de Medicamentos"; "Interações Farmacológicas" & $\begin{array}{l}\text { Pessoas que fazem uso } \\
\text { da TARV e outras } \\
\text { drogas medicamentosas }\end{array}$ \\
\hline $\mathrm{C}$ & Não se aplica & Não se aplica & Não se aplica \\
\hline $\mathrm{O}$ & "Prevalência" & "Coeficiente de Prevalência"; "Taxa de Prevalência" & Ocorrência de IM \\
\hline $\mathrm{T}$ & $\begin{array}{l}\text { "Tratamento } \\
\text { Farmacológico" }\end{array}$ & $\begin{array}{l}\text { "Farmacoterapia"; "Quimioterapia"; "Quimiotratamento"; "Terapia com } \\
\text { Drogas"; "Terapia com Fármacos"; "Terapia com Medicamentos"; } \\
\text { "Terapia Farmacológica"; "Terapia Medicamentosa"; "Terapia por } \\
\text { Drogas"; "Tratamento com Drogas"; "Tratamento com Fármacos"; } \\
\text { "Tratamento com Medicamentos"; "Tratamento Medicamentoso" }\end{array}$ & $\begin{array}{l}\text { Medicações utilizados } \\
\text { pelos pacientes com } \\
\text { HIV/AIDS }\end{array}$ \\
\hline
\end{tabular}

Fonte: Autores.

A busca de artigos foi realizada por meio de publicações científicas indexadas nas bases de dados Literatura LatinoAmericana e do Caribe em Ciências da Saúde (LILACS); Medical Literature Analysis and Retrieval System Online (MEDLINE), Biblioteca Virtual en Salud Enfermería (BDENF - Enfermagem) e Índice Bibliográfico Español en Ciencias de la Salud (IBECS), acessadas através da Biblioteca Virtual de Saúde (BVS) no dia 22 de novembro de 2020, visando delimitar a data de artigos publicados.

Foram elegíveis para o estudo artigos disponíveis na íntegra, com textos completos, publicados nos idiomas português, inglês e/ou espanhol, entre os anos de 2010 e 2020 e que responderam à questão norteadora do estudo. Além disso, os critérios considerados para exclusão foram: repetição de um artigo nas bases de dados selecionadas, estudos como: teses, dissertações, editoriais e estudos que não responderam de modo significativo à questão norteadora do estudo.

Com intuito de otimizar a seleção dos artigos foi utilizado o aplicativo Rayyan (QCRI), com a finalidade de tornar mais rápida a triagem inicial de resumos e títulos, auxiliando todo o processo da revisão sistemática (Ouzzani et al., 2016). A seleção se deu de forma cega e independente, por dois pesquisadores, para exclusão de duplicatas e artigos que não atenderem aos critérios da pesquisa. Divergências entre os pesquisadores foram resolvidas por consenso.

Após a seleção dos artigos e leitura dos textos na íntegra, seguiu-se para análise, interpretação dos resultados e elaboração da matriz de síntese, contendo: autor, ano, periódico, local, identificação e quantidade de interações medicamentosas encontradas. Cabe destacar, que esse método visa a extração e organização dos dados, pois resume seus aspectos complexos encontrados e auxilia no processo de categorização (Cunha et al., 2014). 


\section{Resultados}

Após percorrer as etapas metodológicas estabelecidas na revisão integrativa, identificou-se 166 artigos, foram incluídos 16 artigos mediante os critérios estabelecidos. Dentre os quais, quatorze (87,5\%) estavam disponíveis na língua inglesa, um artigo $(6,25 \%)$ na língua portuguesa e um artigo $(6,25 \%)$ na língua espanhola. As etapas do processo metodológico foram descritas em um fluxograma (Figura 1).

Figura 1: Fluxograma das etapas do processo metodológico, 2020-2021.

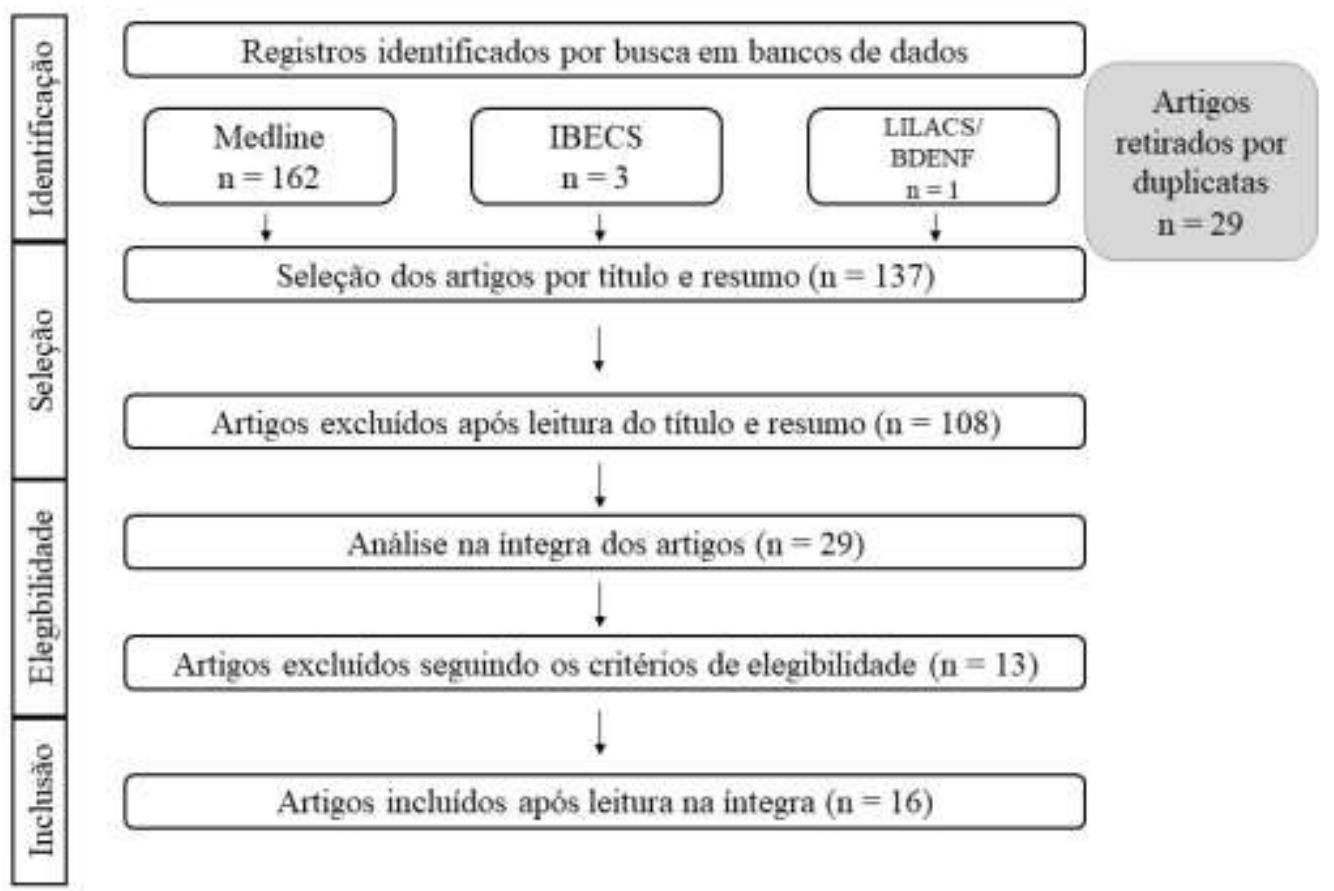

Fonte: Autores.

Mediante os estudos analisados, os artigos incluídos foram realizados em diferentes países, na Espanha foram encontrados 3 artigos, sendo o país com mais estudos, seguido pelo Brasil com 2 artigos selecionados. A faixa etária dos participantes se encontrava entre 16 a 65 anos ou mais. Observou-se que a maior prevalência de IM ocorreu em um estudo no qual identificou-se 730 combinações entre antirretrovirais e medicamentos concomitantes como potencial interação drogadroga (Quadro 1).

Quadro 1. Matriz de síntese com as principais características dos estudos analisados referente às IM, 2010-2020.

\begin{tabular}{|c|c|c|}
\hline AUtOr/AnO/LOCal/Re & Identificação & \\
\hline $\begin{array}{l}\text { BADILLO et al., 2019. Bogotá. } \\
\text { Biomédica. }\end{array}$ & $\begin{array}{l}\text { Pacientes de } 18 \text { anos de idade } \\
\text { ou mais com diagnóstico } \\
\text { confirmado de HIV. }\end{array}$ & $\begin{array}{l}\text { is foram avaliadas, com uma } \\
\text { ente. }\end{array}$ \\
\hline $\begin{array}{l}\text { CANÃBATE, VALÍN, } \\
\text { Espanha. Colômbia médica. }\end{array}$ & $\begin{array}{l}\text { Pacientes com diagnóstico de } \\
\text { HIV+ com } 50 \text { anos ou mais. }\end{array}$ & $\begin{array}{l}\text { antirretroviral e medicamentos não antirretrovi } \\
\text { de } 52 \text { pacientes. }\end{array}$ \\
\hline $\begin{array}{l}\text { RANZANI et al., 2018. Milão. } \\
\text { Journal of Acquired Immune } \\
\text { Deficiency Syndromes (JAIDS). }\end{array}$ & $\mathrm{rac}$ & $\begin{array}{l}\text { A triagem de potenciais IM entre os antirretrovirais e não } \\
\text { antirretrovirais renderam um total de } 694 \text { âmbar-potenciais } \\
\text { interações droga-droga e } 43 \text { vermelhos potenciais interações } \\
\text { droga-droga. }\end{array}$ \\
\hline IEFRIED & Adultos infectados com HIV em & 730 combinações de medicamentos \\
\hline
\end{tabular}




\begin{tabular}{|c|c|c|}
\hline AIDS (London, England). & $\begin{array}{lrrr}\text { TARV e carga } & \text { viral } \\
\text { indetectável. } & & \\
\end{array}$ & $\begin{array}{l}\text { antirretrovirais em } 237 \text { dos } 392 \text { participantes foram identificados } \\
\text { como potencial IM. }\end{array}$ \\
\hline $\begin{array}{l}\text { BASTIDA et al., 2017. Espanha. } \\
\text { Farmacia Hospitalaria. }\end{array}$ & $\begin{array}{l}\text { Pacientes } \geq 65 \text { anos de idade } \\
\text { que receberam tratamento } \\
\text { antirretroviral. }\end{array}$ & Um total de 259 potenciais IM foram detectadas. \\
\hline $\begin{array}{l}\text { PRIYANKA et al., } 2017 . \text { Índia. } \\
\text { International Journal of Risk \& } \\
\text { Safety in Medicine. }\end{array}$ & $\begin{array}{llr}\text { Pacientes com } & \text { HIV } \\
\text { soropositivos acima de } & 18 \text { anos } \\
\text { de idade. } & \end{array}$ & $\begin{array}{l}267 \text { potenciais interações medicamentosas para terapia } \\
\text { antirretroviral foram relatadas em } 107 \text { pacientes. }\end{array}$ \\
\hline $\begin{array}{l}\text { CÓRDOVA et al., 2016. Argentina. } \\
\text { Revista chilena de infectología. }\end{array}$ & 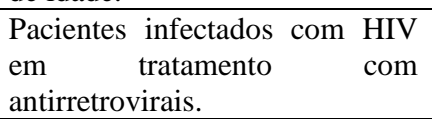 & $\begin{array}{l}\text { Dos pacientes que receberam medicação concomitante, } 68 \\
\text { tinham pelo menos uma interação medicamentosa e apenas três } \\
\text { em associação eram contraindicadas. }\end{array}$ \\
\hline $\begin{array}{l}\text { SANTOS, SECOLI, PADOIN, } 2016 . \\
\text { Brasil. Revista Latino-Americana de } \\
\text { Enfermagem. }\end{array}$ & $\begin{array}{l}\text { Pacientes adultos infectados } \\
\text { com HIV. }\end{array}$ & Foram identificados 218 potenciais interações medicamentosas. \\
\hline $\begin{array}{l}\text { 1., 2015. Uganda. Journal } \\
\text { obial Chemotherapy. }\end{array}$ & $\begin{array}{l}\text { População clínica } \begin{array}{r}\text { adulta que } \\
\text { estavam } \\
\text { antirretrovirais. }\end{array} \\
\end{array}$ & $\begin{array}{l}\text { Um total de } 374 \text { pacientes tinham uma ou mais interações } \\
\text { medicamentosas clinicamente significativas, com } 514 \text { interações } \\
\text { identificadas no total. }\end{array}$ \\
\hline $\begin{array}{l}\text { RAMOS et al., 2015. Brasil. Journal } \\
\text { of Clinical Pharmacy and } \\
\text { Therapeutics }\end{array}$ & $\begin{array}{l}\text { Pacientes com HIV/AIDS que } \\
\text { tinham } 18 \text { anos ou mais. }\end{array}$ & $\begin{array}{l}\text { Das } 106 \text { prescrições analisadas, o Micromedex identificou } 347 \\
\text { interações droga-droga em potencial e o Drugs.com identificou } \\
615 \text { interações droga-droga potenciais. Um total de } 307 \\
\text { interações droga-droga foram descritos em ambos os bancos de } \\
\text { dados. }\end{array}$ \\
\hline $\begin{array}{l}\text { INIESTA-NAVALÓN et al., } 2015 . \\
\text { Espanha. Hiv Medicine. }\end{array}$ & $\begin{array}{l}\text { Pacientes com } 18 \text { anos ou mais } \\
\text { infectados com HIV. }\end{array}$ & $\begin{array}{l}\text { Um total de } 292 \text { potenciais interações medicamentosas foram } \\
\text { identificadas, dos quais } 102 \text { eram interações medicamentosas } \\
\text { clinicamente significativas. }\end{array}$ \\
\hline $\begin{array}{l}\text { HOLTZMAN et al., 2013. EUA. } \\
\text { Journal of general internal medicine. }\end{array}$ & Adultos infectados & $\begin{array}{l}\text { mais combinações entre } \\
\text { n contraindicadas. }\end{array}$ \\
\hline $\begin{array}{l}\text { MARZOLI et al., 2011. Suiça. } \\
\begin{array}{l}\text { Journal of } \\
\text { chemotherapy. }\end{array}\end{array}$ & $\begin{array}{l}\text { Pacientes tratados com ART } \\
\text { agrupados como pacientes mais } \\
\text { velhos }(\geq 50 \text { anos }) \text { e pacientes } \\
\text { mais jovens }(<50 \text { anos }) \text {. }\end{array}$ & Quantidade de interações medicamentosas não informadas. \\
\hline $\begin{array}{l}\text { HASAN et al., 2011. Malásia. } \\
\text { Medical principles and practice. }\end{array}$ & $\begin{array}{l}\text { Pacientes com HIV/AIDS com } \\
\text { idade média de } 22,94 \text { anos. }\end{array}$ & $\mathrm{Qu}$ \\
\hline $\begin{array}{l}\text { KIGEN et al., 2011. Quênia. PloS } \\
\text { one. }\end{array}$ & $\begin{array}{l}\text { Paciente soropositivos para HIV } \\
\text { com idade }>16 \text { anos recebendo } \\
\text { terapia antirretroviral. }\end{array}$ & $\begin{array}{l}\text { O risco de interações clinicamente significativas foi identificado } \\
\text { em } 334 \text { pacientes, com grandes interações em } 147 \text { pacientes. }\end{array}$ \\
\hline $\begin{array}{l}\text { MARZOLI et al., 2010. Suíça. } \\
\text { Antiviral therapy. }\end{array}$ & $\begin{array}{l}\text { Indivíduos infectados pelo HIV } \\
\text { com idade } \geq 16 \text { anos }\end{array}$ & $\begin{array}{l}\text { No geral, } 40 \% \text { dos pacientes tiveram } \geq 1 \text { potenciais interações } \\
\text { droga-droga. Um máximo de } 11 \text { potenciais interações droga- } \\
\text { droga foi observada em dois indivíduos. }\end{array}$ \\
\hline
\end{tabular}

Fonte: Autores, por meio dos artigos analisados.

A partir da análise dos artigos, foi possível identificar as principais combinações de medicamentos da TARV e outras terapias utilizadas, que foram sinalizadas como potenciais IM nos participantes estudados. A título de otimização da tabela as IM que apresentaram apenas uma ocorrência foram classificadas como “Outros” (Tabela 1). 
Tabela 2. Medicações encontradas em IM nos estudos analisados, 2010-2020.

\begin{tabular}{lcc}
\hline \multicolumn{1}{c}{ Combinação de interações medicamentosas } & Ocorrência de IM nos Estudos & \% \\
& $\mathbf{n}^{\circ}$ & 2,72 \\
Atazanavir com esomeprazol & 3 & 2,72 \\
Atazanavir com omeprazol & 3 & 1,82 \\
Atazanavir com pantoprazol & 2 & 2,72 \\
Atazanavir com ranitidina & 3 & 1,82 \\
Efavirenz com midazolam & 2 & 1,82 \\
Efavirenz com rifampicina & 2 & 1,82 \\
Nelfinavir com esomeprazol & 2 & 1,82 \\
Nevirapina com rifampicina & 2 & 1,82 \\
Rilpivirina com omeprazol & 2 & 1,82 \\
Ritonavir com atorvastatina & 2 & 1,82 \\
Ritonavir com fluconazol & 2 & 1,82 \\
Ritonavir com quetiapina & 2 & 1,82 \\
Tenofovir com aciclovir & 2 & 1,82 \\
Zidovudina com dipirona & 2 & 71,82 \\
Outros & 2 & 100,00 \\
Total & 79 & 110 \\
\hline
\end{tabular}

Fonte: Autores, por meio dos artigos analisados.

\section{Discussão}

Mediantes os resultados encontrados, foi possível identificar que as pesquisas referentes a IM em pacientes com HIV/AIDS, são realizadas em diversas faixas etárias, sendo mais frequente em adultos. Cabe ressaltar, que todos os pacientes com esse diagnóstico e em tratamento apresentam o risco de IM por utilizar grande quantidade de medicamentos. Porém, pacientes idosos com HIV/AIDS podem exigir mais medicamentos para tratar também comorbidades existentes, além da TARV que geralmente consiste em combinações de em média três antirretrovirais (RANZANI et al., 2018). Evidências apontam que idosos possuem 51\% de chance de apresentar IM em relação aos jovens, se realizar o uso de 6 ou 7 medicamentos (Santos et al., 2016).

Dessarte, o manejo da infecção pelo HIV em pacientes mais velhos pode apresentar complicações devido a ocorrência de polifarmácia e consequente elevação da taxa de IM, uma vez que, com o uso medicamentos combinados com a TARV verifica-se a possibilidade de elevação da toxicidade das drogas utilizadas (Marzolini et al., 2011).Por meio dos estudos analisados, percebe-se que foram desenvolvidas apenas três pesquisas com inclusão da população idosa acometida com o HIV (Fernández Cañabate \& Ortega Valín, 2019; Bastida et al., 2017; Marzolini et al., 2011)., tornando difícil quantificar e identificar quais intervenções poderiam ser realizadas visando prevenir as IM e suas complicações.

Além disso, o estudo com esta população é necessário pelo fato do aparecimento de mais comorbidades com o envelhecimento, o que eleva o uso de fármacos com ou sem prescrição médica, aumentando o risco para potenciais IM e interações clinicamente significativas.

Em relação aos medicamentos utilizados pelos pacientes com diagnóstico de HIV/AIDS observou-se que são utilizadas combinações de drogas para tratar outras doenças, efeitos colaterais e eventos adversos. Segundo SIEFRIED et al. (2018), até dois terços dos pacientes com HIV/AIDS utilizam algum medicamento concomitante para amenizar a TARV, reduzir os efeitos colaterais e tratar comorbidades. Assim, essa combinação de medicações pode complicar os cuidados com o HIV, contribuir para a polifarmácia, IM, efeitos colaterais e baixa adesão ao tratamento.

Os principais medicamentos utilizados por pacientes com HIV/AIDS envolvidos nas IM são os inibidores de protease, inibidores de transcriptase reversa não nucleosídeo e inibidores de transcriptase reversa nucleosídeo associados principalmente a medicamentos inibidores da bomba de prótons. Conforme PONTELO et al. (2020) os medicamentos coadministrados e o uso de inibidores de protease foram identificados como fatores de risco, uma vez que predispõe o aumento de medicamentos 
coadministrados, com a utilização de regimes contendo inibidores de protease houve um aumento na possibilidade de desenvolver IM. Além disso, a inclusão de um medicamento elevou a chance de IM em 12,9\% e a inclusão de inibidores de protease no regime de antirretrovirais aumentou a probabilidade de IM em $10 \%$.

Ao realizar a análise das IM no site Medscape (2021), por meio do Drug Interaction Checker, foram encontradas IM entre atazanavir, darunavir e ritonavir (inibidores de protease), associados principalmente a medicações como a sinvastatina, fenobarbital e omeprazol. Os medicamentos coadministrados juntamente com TARV, ocasionaram a diminuição do efeito dos antirretrovirais ao afetar o metabolismo da enzima hepática ou intestinal, resultando na perda do efeito terapêutico ou em resistência ao darunavir. Em relação a sinvastatina associada a TARV, foi detectada um aumento do nível ou efeito e até mesmo a toxicidade da mesma. Ao mesmo tempo, em que houve um aumento dos níveis ou efeito do ritonavir.

Ademais, o uso de medicamentos como atazanavir ou rilpirivina em combinação com omeprazol podem acarretar redução de concentração correspondente ao aumento do pH gástrico causado pelo omeprazol, reduzindo a absorção do antirretroviral e aumentando o risco de uma falha virológica (Fernández Cañabate \& Ortega Valín, 2019).

Ressalta-se que, durante a análise dos artigos outras classes de medicamentos também foram envolvidas nas combinações de IM, como benzodiazepínicos, estatinas, antipsicóticos, antifúngicos, antibióticos e entre outros. Essas combinações com outros medicamentos e antirretrovirais pode resultar em mudanças importantes nos níveis séricos dos medicamentos administrados e diversas vezes estão relacionadas a eventos adversos evitáveis. Há evidências que somente a TARV ou a combinação com outros medicamentos, pode apresentar ação no Sistema Nervoso Central, alterações metabólicas, entre outras (Santos et al., 2016).

Cabe destacar, a ocorrência de IM pode apresentar complicações no tratamento do paciente dependendo da gravidade, que pode permear entre grave, moderada e leve a depender da base de dados utilizada para essa avaliação. Dessa forma, é fundamental que intervenções sejam realizadas precocemente visando evitar consequências para o paciente. De acordo com Iniesta-Navalón et al. (2015), conhecer e gerenciar as IM é fundamental visando a otimização da assistência aos pacientes com HIV/AIDS, ajudando a prevenir eventos adversos e perda de eficácia dos medicamentos administrados.

Este estudo apresentou algumas limitações como, dificuldade em estimar a prevalência de IM em paciente com HIV/AIDS. Por se tratar de uma análise por meio de estudos científicos, nota-se que a ocorrência de IM ou de suas complicações nesta população ainda é pouco estudada. Além disso, poucos estudos foram desenvolvidos no Brasil e dessa forma não é possível determinar o perfil de IM no país e as intervenções realizadas no país mediante a presença de IM, colocando em risco a segurança dos pacientes dessa determinada população. Ressalta-se que para isto, é necessário que a segurança do paciente seja prioridade em todos os níveis de atenção, com profissionais capazes de disseminar uma cultura de segurança pactuada com a gestão e com subsídios capazes de evitar eventos adversos desde a análise da prescrição medicamentosa, assegurando a continuidade e a segurança da terapia medicamentosa, favorecendo de forma eficiente a segurança do paciente e garantindo uma melhor qualidade na assistência (Etelvino et al., 2019).

Destarte, poucos estudos relacionam a enfermagem e sua atuação diante a associação de medicamentos utilizados pelo paciente com HIV/AIDS e o potencial risco de IM durante o tratamento. Ademais, percebe-se ausência de estudos que relatam a atuação da equipe multidisciplinar no cuidado integral a esses indivíduos, permitido assim, a identificação precoce de IM.

\section{Conclusão}

As evidências comprovam que a ocorrência de IM é frequente em pacientes com diagnóstico de HIV/AIDS, principalmente na população idosa. Isso ocorre, pois, esses pacientes utilizam diversos medicamentos além dos antirretrovirais, sendo eles para o tratamento de comorbidades, efeitos colaterais e eventos adversos, o que eleva o risco de IM. Além disso, 
estudos apontaram que grande parte das interações ocorreram entre medicamentos antirretrovirais associados a inibidores da bomba de prótons, benzodiazepínicos, antipsicóticos, antibióticos e estatinas.

Entretanto, essa temática ainda é pouco estudada mundialmente. Sendo assim, a partir deste estudo, sugere-se novas pesquisas sobre o assunto, buscando conhecer o perfil dos pacientes que apresentam IM, quais as principais medicações inclusas nas interações droga-droga, quais as intervenções devem ser realizadas, visando a formulação de estratégias que busquem a minimização de riscos para os pacientes atendidos.

Como forma de minimizar o problema é fundamental que haja uma comunicação efetiva e integrada entre os membros da equipe multidisciplinar que prestam assistência a esses pacientes, para a evidenciação precoce de IM, evitando as complicações, eventos adversos e efeitos colaterais, a redução de hospitalizações e qualidade de assistência prestada aos pacientes. A atuação da enfermagem se torna essencial na detecção do risco de IM durante o tratamento, uma vez que o enfermeiro está inserido de diretamente na prestação do cuidado aos pacientes com HIV/AIDS.

\section{Referências}

Andrade, K. V. F., \& Souza, A. M. (2018). Prevalência de interações medicamentosas potenciais em indivíduos hipertensos acompanhados na estratégia de saúde da família. Journal of Health \& Biological Sciences, 6(4), 405-411.

Antunes, J. D. F. S., Okuno, M. F. P., Lopes, M. C. B. T., Campanharo, C. R. V., \& Batista, R. E. A. (2015). Interação medicamentosa em idosos internados no serviço de emergência de um hospital universitário. Revista Mineira de Enfermagem, 19(4), 907-918.

Baecke, C., Gyssens, I. C., Decoutere, L., van der Hilst, J. C. H., \& Messiaen, P. (2017). Prevalence of drug-drug interactions in the era of HIV integrase inhibitors: a retrospective clinical study. Neth J Med, 75(6), 235-240.

Bastida, C., Grau, A., Márquez, M., Tuset, M., De Lazzari, E., Martínez, E., \& Gatell, J. M. (2017). Polypharmacy and potential drug-drug interactions in an HIV-infected elderly population. Farmacia Hospitalaria, 41(5), 618-624.

Bouzillé, G., Morival, C., Westerlynck, R., Lemordant, P., Chazard, E., Lecorre, P., .\& Cuggia, M. (2019, August). An Automated Detection System of DrugDrug Interactions from Electronic Patient Records Using Big Data Analytics. In MedInfo (pp. 45-49).

Carvalho, B. M. D., Morandi, M. S., Parro, M. C., \& Moreno, A. D. H. (2016). Avaliação de prescrições e análise da ocorrência de interações medicamentosas. CuidArte, Enferm, 123-130.

Costa, D. F., Gonçalves, A. S. R., dos Santos Vieira, J. R., \& Guerreiro, J. F. (2018). Adesão à terapia antirretroviral de pacientes portadores de HIV/Aids com lipodistrofia. Revista Enfermagem UERJ, 26, 31156.

Cunha, P. L. P., Cunha, C. S., \& Alves, P. F. (2014). Manual Revisão Sistemática Integrativa: a pesquisa baseada em evidências. Belo Horizonte, 63.

Etelvino, M. A. L., dos Santos, N. D., Aguiar, B. G. C., \& de Assis, T. G. (2019). Segurança do paciente: uma análise do aprazamento de medicamentos. Enfermagem em Foco, 10(4).

Gervasoni, C., Formenti, T., \& Cattaneo, D. (2019). Management of polypharmacy and drug-drug interactions in HIV patients: a 2-year experience of a multidisciplinary outpatient clinic. AIDS reviews, 21(1), 40-49.

Hasler, S., Senn, O., Rosemann, T., \& Neuner-Jehle, S. (2015). Effect of a patient-centered drug review on polypharmacy in primary care patients: study protocol for a cluster-randomized controlled trial. Trials, 16(1), 1-7.

Iniesta-Navalón, C., Franco-Miguel, J. J., Gascón-Cánovas, J. J., \& Rentero-Redondo, L. (2015). Identification of potential clinically significant drug interactions in HIV-infected patients: a comprehensive therapeutic approach. HIV medicine, 16(5), 273-279.

Joaquim, F. L., Silva, R. M. C. R. A., Garcia-Caro, M. P., Cruz-Quintana, F., \& Pereira, E. R. (2018). Impact of venous ulcers on patients' quality of life: an integrative review. Revista brasileira de enfermagem, 71, 2021-2029.

Kara, E., İnkaya, A. Ç., Hakli, D. A., Demirkan, S. K., \& Ünal, S. (2019). Polypharmacy and drug-related problems among people living with HIV/AIDS: a single-center experience. Turkish journal of medical sciences, 49(1), 222-229.

Marzolini, C., Back, D., Weber, R., Furrer, H., Cavassini, M., Calmy, A., \& Yerly, S. (2011). Ageing with HIV: medication use and risk for potential drugdrug interactions. Journal of antimicrobial chemotherapy, 66(9), 2107-2111.

Fernández Cañabate, S., \& Ortega Valín, L. (2019). Polypharmacy among HIV infected people aged 50 years or older. Colombia Médica, 50(3), 142-152.

Medscape. Drug Interaction Checker. (2021) https://reference.medscape.com/drug-interactionchecker

Monteiro, C. R. D. A., Schoueri, J. H. M., Cardial, D. T., Linhares, L. D. C., Turke, K. C., Steuer, L. V., \& Giglio, A. D. (2019). Evaluation of the systemic and therapeutic repercussions caused by drug interactions in oncology patients. Revista da Associação Médica Brasileira, 65, 611-617. 
Monteiro, S. C. M., Belfort, I. K. P., Sousal, W. R., Barros, C. S., \& Campos, K. V. S. (2015). Estudo de potenciais interações medicamentosas em pacientes hipertensos. Infarma Ciências Farmacêuticas, 27(2), 117-25.

Moreira, M. B., Mesquita, M. G. D. R., Stipp, M. A. C., \& Paes, G. O. (2017). Potential intravenous drug interactions in intensive care. Revista da Escola de Enfermagem da USP, 51 .

Nunes Júnior, S. S., \& Ciosak, S. I. (2018). Terapia antirretroviral para HIV/AIDS: o estado da arte. Rev. enferm. UFPE on line, $1103-1111$.

Osorio, L., Rivera, M., Pino-Marín, D. E., Giraldo, N. A., \& Amariles, P. (2019). Relevancia clínica de las interacciones medicamentosas en pacientes infectados con el virus de la inmunodeficiencia humana: actualización 2015-2017. Revista chilena de infectología, 36(4), 475-489.

Ouzzani, M., Hammady, H., Fedorowicz, Z., \& Elmagarmid, A. (2016). Rayyan—a web and mobile app for systematic reviews. Systematic reviews, 5(1), 110 .

Pontelo, B. M., Greco, D. B., Guimarães, N. S., Rotsen, N., Braga, V. A. R., Pimentel, P. H. N., ... \& Tupinambás, U. (2020). Profile of drug-drug interactions and impact on the effectiveness of antiretroviral therapy among patients living with HIV followed at an Infectious Diseases Referral Center in Belo Horizonte, Brazil. Brazilian Journal of Infectious Diseases, 24, 104-109.

Popets, J. C., Andrade, G. B., Stroka, A., \& Rostelato-Ferreira, S. (2015). Estudo das interações medicamentosas em prescrições de pacientes de uma ClínicaEscola de Fisioterapia. Infarma-Ciências Farmacêuticas, São Paulo, 28(1), 22-26.

Ranzani, A., Oreni, L., Agrò, M., van den Bogaart, L., Milazzo, L., Giacomelli, A., \& Ridolfo, A. L. (2018). Burden of exposure to potential interactions between antiretroviral and non-antiretroviral medications in a population of HIV-positive patients aged 50 years or older. JAIDS Journal of Acquired Immune Deficiency Syndromes, 78(2), 193-201.

Rodrigues, S. B., Louro, D., Souza, E. M. O., \& da Cunha, M. A. (2020). Estabelecimento da comunicação terapêutica entre enfermeiro e clientes frente a tentativas de autoextermínio: revisão integrativa da literatura. Brazilian Journal of Health Review, 3(3), 5943-5958.

Santos, W. M., Secoli, S. R., \& de Mello Padoin, S. M. (2016). Potenciais interações de drogas em pacientes de terapia antirretroviral. Revista LatinoAmericana de Enfermagem, 24, 1-9.

Siefried, K. J., Mao, L., Cysique, L. A., Rule, J., Giles, M. L., Smith, D. E., ... \& PAART study investigators. (2018). Concomitant medication polypharmacy, interactions and imperfect adherence are common in Australian adults on suppressive antiretroviral therapy. AIDS (London, England), 32(1), 35.

Silva, H. F. N., Dourado, C. S. D. M. E., Silva, H. G. N., \& Silva, H. F. N. (2019). Avaliação do tratamento antirretroviral de pessoas convivendo com HIV/aids que participam de um grupo de adesão. Medicina (Ribeirao Preto), 52(3), 161-170.

Smit, M., Brinkman, K., Geerlings, S., Smit, C., Thyagarajan, K., van Sighem, A., \& Hallett, T. B. (2015). Future challenges for clinical care of an ageing population infected with HIV: a modelling study. The Lancet Infectious Diseases, 15(7), 810-818.

Sousa, L. M. M., Marques-Vieira, C. M. A., Severino, S. S. P., \& Antunes, A. V. (2017). A metodologia de revisão integrativa da literatura em enfermagem. $N^{o} 21$ Série 2-Novembro 2017, 17.

Stader, F., Kinvig, H., Battegay, M., Khoo, S., Owen, A., Siccardi, M., \& Marzolini, C. (2018). Analysis of clinical drug-drug interaction data to predict magnitudes of uncharacterized interactions between antiretroviral drugs and comedications. Antimicrobial agents and chemotherapy, 62(7), e00717-18.

Tavares, D. S., Gomes, N. C., Rodriguês, L. R., \& Tavares, D. M. D. S. (2018). Perfil de idosos com síndrome metabólica e fatores as sociados às possíveis interações medicamentosas. Revista Brasileira de Geriatria e Gerontologia, 21, 164-175.

Turgeon, J., \& Michaud, V. (2016). Clinical decision support systems: Great promises for better management of patients' drug therapy. Expert opinion on drug metabolism \& toxicology, 12(9), 993-995. 